\title{
DELAMINATION GROWTH ANALYSIS IN MODE I BY MEANS OF LAMB WAVES
}

\author{
Lenka Michalcová ${ }^{1}$ \\ 1 Aerospace Research and Test establishment, Strength of Structures Department, Beranových 130, \\ 19905, Prague - Letňany, Czech Republic. E-mail: michalcova@vzlu.cz
}

\section{Introduction}

Guided wave based monitoring of composite structures plays an important role in the area of structural health monitoring (SHM) of aerospace structures [1, 2]. Delamination growth of double cantilever beam specimens (DCB) using Lamb waves is investigated from the point of frequency tuning, actuation waveform, sensor placement and selection of parameter sensitive to delamination growth [3].

\section{Materials and methods}

Specimens were made of carbon fiber plain weave fabric reinforced epoxy resin with a sequence of lamination $[45 / 0]_{4 s}$. DCB tests were performed according to the ASTM D5528 standard. A polymer foil with the length of approximately $25 \mathrm{~mm}$ was inserted into the midplane of the laminated panels as an artificial initial delamination (Fig. 1).

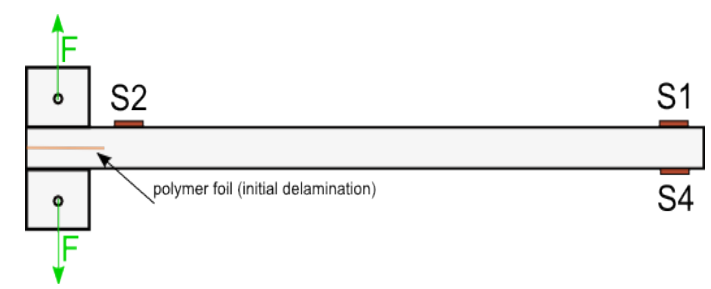

Fig. 1 Scheme of DCB specimen

Mechanical tests were performed on INOVA ZUZ-50 and a load cell with capacity of $1 \mathrm{kN}$ was used. Acellent Scan-genie II system with 3 smart layers sensors was utilized for periodical guided wave monitoring.

The objective of this work is to compare visually based and Lamb wave based method of crack growth curve determination.

\subsection{Selection of actuating parameters for Lamb wave measurements}

Lamb waves propagate only in plate-like structures and propagating modes - symmetric and asymmetric (Fig. 2) - are dependent on actuating

frequency and structure thickness. Pitch-catch configuration with transducer S2 as the actuator and S1 and S4 as sensors was utilized. Parameters suitable for signal actuation were selected experimentally before the test. Fundamental A0 mode propagated without any interference with other modes in the frequency range of $30-50 \mathrm{kHz}$. Burst 5 (a sine wave of 5 cycles) actuation pulse with the frequency of $40 \mathrm{kHz}$ and applied voltage of $50 \mathrm{~V}$ and $20 \mathrm{~dB}$ amplification was set as optimal.

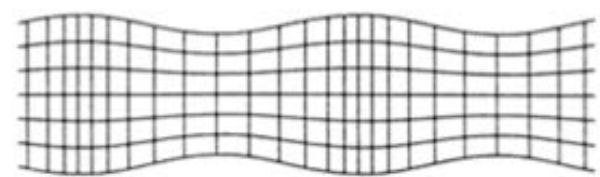

Symmetric mode

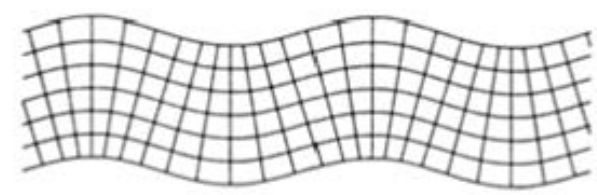

Asymmetric mode

Fig. 2 Lamb wave modes

\subsection{Parameter sensitive to delamination growth}

Group velocity of Lamb waves is dependent on the product of frequency and thickness. These relations in general describe dispersion curves. With delamination growth the part of the specimen with half thickness extends which results in time delays of signal arrivals compared to the baseline measurement. 


\section{Results}

Example of signal delay and resulting signals plot from all measurements (10 measurements as the delamination propagated) are shown in Fig. 3. The first arrival time shift curve was highlighted on order to show the trend of time delays and delamination length.

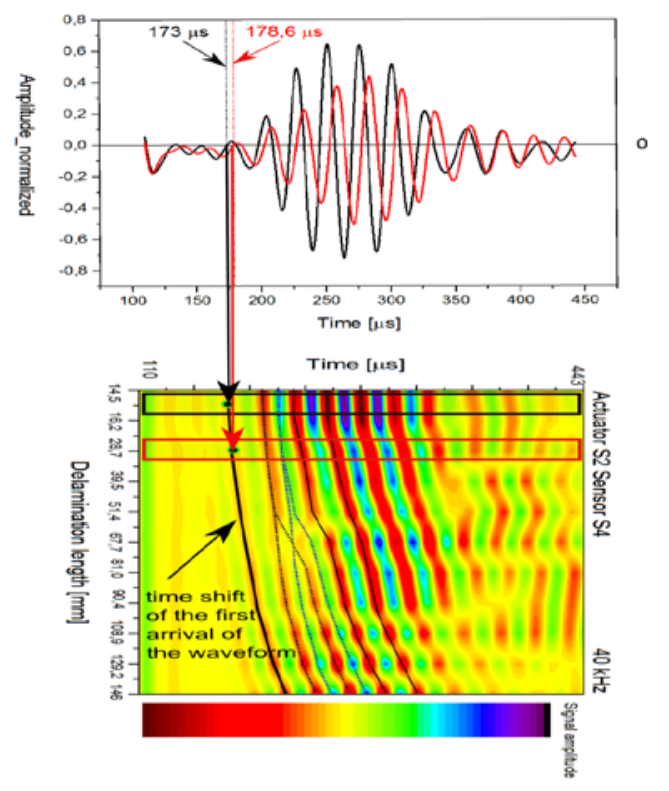

Fig. 3 Time shift of the first arrival of the waveform

Time shifts of the first signal arrival compared to the baseline measurement was utilized for the exact crack tip localization according to the following equation:

$$
a_{n}=\frac{v_{1} v_{2} d t_{n}}{v_{2}-v_{1}},
$$

where $a_{n}$ represents the crack length, $v_{1}$ and $v_{2}$ represent group velocity of $40 \mathrm{kHz}$ Lamb wave in the specimen with half of thickness and full thickness respectively and $d t_{n}$ denotes the time difference of the $n$th measurement compared to the baseline state.

The knowledge of both velocities is conditional. Velocity $v_{1}$ was determined using the Eq. (1) with one example of visually identified crack length and corresponding $d t_{n}$.

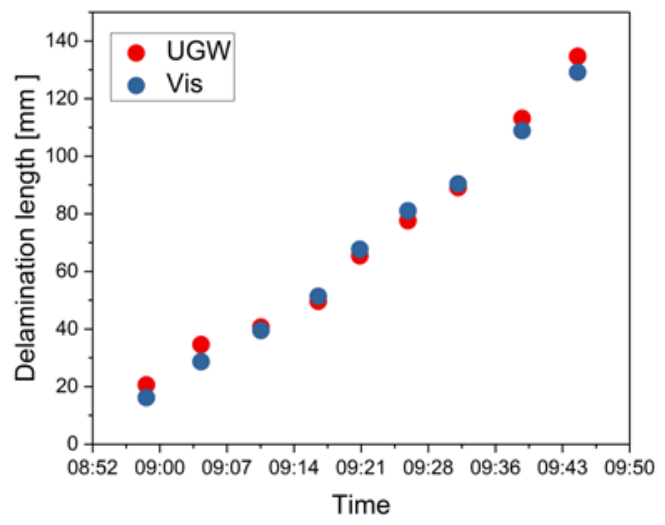

Fig. 4 Comparison of visually based and Lamb wave based delamination length

\section{Conclusion}

To conclude, Lamb wave monitoring of delamination growth showed great agreement with visually based data. However, from the point of view of SHM approach the velocity of lamb waves in the delaminated specimen should not be determined using visually identified delamination length since this supposed to be the output. The velocity should be determined before the test e.g. by dispersion curves calculation.

\section{Acknowledgements}

This work was funded by the Ministry of Industry and Trade of the Czech Republic in the framework of Institutional support of Research organizations.

\section{References}

[1] Rose, J.L., Ultrasonic Guided Waves in Solid Media. Elsevier, 2014.

[2] Giurgiutiu, V., Structural Health Monitoring with Piezoelectric Wafer Active Sensors. Cambridge University Press, 2008.

[3] Ramadas, C., Hood, A., Balasubramaniam, K., Joshi, K., Ultrasonic Lamb Wave Based Crack Growth Prediction for Estimation of Strain Energy Release Rate, editors B.S.S. Daniel, and G.P. Chaudhari, Advanced Materials Research, 2012. pp. 24-28. 